\title{
Paper Linear and Planar Array Pattern Nulling via Compressed Sensing
}

\author{
Jafar Ramadhan Mohammed ${ }^{1}$, Raad H. Thaher ${ }^{2}$, and Ahmed Jameel Abdulqader ${ }^{1,2}$, \\ ${ }^{1}$ College of Electronic Engineering, Ninevah University, Mosul, Iraq \\ ${ }^{2}$ College of Engineering, Mustansiriyah University, Baghdad, Iraq
}

https://doi.org/10.26636/jtit.2021.152921

\begin{abstract}
An optimization method based on compressed sensing is proposed for uniformly excited linear or planar antenna arrays to perturb excitation of the minimum number of array elements in such a way that the required number of nulls is obtained. First, the spares theory is relied upon to formulate the problem and then the convex optimization approach is adopted to find the optimum solution. The optimization process is further developed by using iterative re-weighted $l_{1}$ norm minimization, helping select the least number of the sparse elements and impose the required constraints on the array radiation pattern. Furthermore, the nulls generated are wide enough to cancel a whole specific sidelobe. Simulation results demonstrate the effectiveness of the proposed method and the required nulls are placed with a minimum number of perturbed elements. Thus, in practical implementations of the proposed method, a highly limited number of attenuators and phase shifters is required compared to other, conventional methods.
\end{abstract}

Keywords-compressed sensing, convex optimization, iterative re-weighted $l_{1}$ - norm minimization, linear and planar arrays.

\section{Introduction}

One of the challenges in current and future wireless communication systems is the presence of interfering signals that may originate either from pre-specified and known or from unknown directions. In such cases, performance of the system may be significantly degraded. This problem becomes more significant in such applications as satellites [1], fifth-generation wireless communications [2] and modern radars [3], as the system of this type are usually expected to operate in environments characterized by sever interference and a very crowded spectrum. One of the simplest and most powerful techniques for eliminating these interfering signals is to point the nulls of the array radiation pattern in the direction of the unwanted interfering signals.

Null placement may be achieved by accurately controlling such array design variables such as element excitation weights and element spacing [4], [5]. Conventionally, all the weights and/or positions of the array elements were perturbed to place the required nulls. Thus, the final phased array systems were usually complex, slow in their convergences, and expensive [6]. Many researchers have investigated the complexity of such fully perturbed antenna arrays, coming up with some solutions. Some of them suggest simple deterministic approaches, such as iterative Fourier transform method [7] and the edge-element method [8], [9] to identify those elements that need to be perturbed for achieving the required null placement. Other scientists, meanwhile, used numerical optimization algorithms, such as the genetic algorithm [4], [10], particle swarm optimization [11], simulated annealing [12], evolutionary algorithms [13], adaptive algorithms [14], cuckoo search optimization [15], invasive weeds optimization [16], and grey wolf optimization [17], to optimize the excitations of the perturbed elements. None of the aforementioned techniques offers a clear path towards selecting the minimum required number of perturbed elements needed in order to place the required number of nulls. In fact, they always assumed that the number of the perturbed elements should be higher than the total number of the required nulls in order to insure an accurate pattern nulling capability. Thus, the number of the perturbed elements was excessive and the solutions were usually not optimal.

Other methods include the use of clustered arrays in which the main arrays were divided into clusters that may consist of either regular or irregular clustered elements [18]-[20]. Furthermore, paper [21] suggested a partially thinned array approach that was applied to side elements only, thus creating a relatively low complexity null placement method. In addition, the structure of a conventional adaptive sidelobe canceller system used in spaced radars has been greatly simplified by using different auxiliary configurations [22] in order to create another solution to this important issue. In light of the above discussions, there is a great need for a new optimized method that is capable of perturbing only the exactly required number of elements in order to place the required number of nulls in an efficient manner. In a bid to solve the problem, compressed sensing was suggested in [23], [24] in order to significantly reduce complexity of array feeding networks. In [25], Bayesian compressed sensing was suggested to find the best match between the sparse array and the reference patterns. Generally, several sparse recovery algorithms exist, such as Yalli [26], smoothed $l_{0}$-norm [27], orthogonal matching pursuit [28], and iterative hard threshold [29] that may be used to solve 
the complexity problem and achieve the desired patterns. Some of these algorithms, like Yalli and smoothed $l_{0}$-norm, usually do not accurately recover the sparse solutions. On the other hand, the iterative reweighted $l_{1}$-norm [30] and the two-steps $l_{0}$ [31] methods were used to efficiently determine the minimum number of perturbed elements and to achieve the desired constraints.

In this paper, radiation patterns of linear and planar arrays are optimized by means of the compressed sensing approach, making sure that the required nulls are placed under a minimum number of perturbed elements. First, the sparse recovery array is built, and then it is implemented with convex optimization applied in order to find the optimum solution. The sparsity of the solution is enhanced through the use of the iterative reweighted $l_{1}$-norm algorithm [32]. That approach allowed the required nulls to be placed efficiently with precisely the needed number of perturbed elements.

\section{Principles of the Method}

For simplicity, consider a linear array of $N$ isotropic elements in which the array pattern may be expressed by:

$$
A F_{\text {uniform }}(\theta)=\sum_{n=1}^{N} x_{\text {on }} \mathrm{e}^{\mathrm{j} k d_{n} u},
$$

where $\theta$ is the observation angle around the array axis, $k=\frac{2 \pi}{\lambda}$ is the wave number, $\lambda$ is the wavelength, $u=\sin \theta$, $d_{n}$ is the position of elements along the $x$ axis which is represented by $d_{n}=\left(n-\frac{N+1}{2}\right) d$, and $d$ is the element spacing. Further, $x_{o n}=a_{o n} \mathrm{e}^{-\mathrm{j} k d_{n} u_{o}}$, where $a_{o n}$ is the array amplitude, $u_{o}=\sin \theta_{o}$, and $\theta_{o}$ is the steering angle of the main beam. After substitution, Eq. (1) can be rewritten as:

$$
A F_{\text {uniform }}(\theta)=\sum_{n=1}^{N} a_{o n} \mathrm{e}^{\mathrm{j} k d_{n}\left(u-u_{o}\right)}
$$

To place a number of wide nulls equal to $Q$, where $q=1,2, \ldots, Q$, we need to perturb the element weights as follows:

$$
X_{n}=x_{o n}+x_{n},
$$

where $x_{n}$ is the weight of the sparse elements. The array factor at the null directions is equal to zero, $A F\left(\theta_{q}\right)=0$. Then Eq. (2) can be modified accordingly:

$$
A F\left(\theta_{q}\right)=\sum_{n=1}^{N} X_{n} \mathrm{e}^{\mathrm{j} k d_{n} u_{q}}=\sum_{n=1}^{N}\left(x_{o n}+x_{n}\right) \mathrm{e}^{\mathrm{j} k d_{n} u_{q}},
$$

which can be rewritten as:

$$
A F_{\text {uniform }}\left(\theta_{q}\right)=-\sum_{n=1}^{N} x_{n} \mathrm{e}^{\mathrm{j} k d_{n} u_{q}} .
$$

This is a set of linear equations that can be written as $\boldsymbol{A x}=\boldsymbol{b}$ where $\boldsymbol{A}=\sum_{n=1}^{N} \mathrm{e}^{j k d_{n} u_{q}}, \boldsymbol{x}=x_{n}$, and $\boldsymbol{b}=-A F_{\text {uniform }}\left(\theta_{q}\right)$. Note that vector $\boldsymbol{x}$ with size $N \times 1$ is the sparse weight that needs to be found and it contains both zero and non-zero values. Vector $\boldsymbol{b}$ with size $Q \times 1$ is the magnitude of the uniform array pattern at null directions with the opposite phase and, finally, $\boldsymbol{A}$ is the matrix with size $Q \times N$. These three parameters can be written as:

$$
\begin{gathered}
\boldsymbol{x}=\left[x_{1}, x_{2}, \ldots x_{N}\right]^{T}, \\
\boldsymbol{b}=\left[-A F_{\text {uniform }}\left(\theta_{1}\right),-A F_{\text {uniform }}\left(\theta_{2}\right), \ldots,\right. \\
\\
\left.-A F_{\text {uniform }}\left(\theta_{Q}\right)\right]^{T}, \\
\boldsymbol{A}=\left[\begin{array}{ccc}
\mathrm{e}^{-\mathrm{j} k d_{1} u_{1}} & \cdots & \mathrm{e}^{-\mathrm{j} k d_{N} u_{1}} \\
\vdots & \ddots & \vdots \\
\mathrm{e}^{-\mathrm{j} k d_{1} u_{Q}} & \cdots & \mathrm{e}^{-\mathrm{j} k d_{N} u_{Q}}
\end{array}\right]
\end{gathered}
$$

Suppose that the number of array elements is larger than the number of the required nulls, $Q<N$, which is the practical scenario, especially for large arrays that consist of hundreds of elements. In such a case, the system will have infinite solutions. To find the minimum number of the perturbed elements, Eq. (8) can be solved using the $l_{o}$ norm minimization approach. However, the problem becomes non-convex and cannot be solved by means of convex optimization. Thus, it is first converted to the convex type by using the $l_{1}$ norm that can be solved by:

$$
\text { minimize }\|\boldsymbol{x}\|_{1} \text { subject to } \boldsymbol{A x}-\boldsymbol{b} \leq \boldsymbol{\varepsilon} .
$$

This equation can be solved by using the convex optimization approach, with its implementation explained in [33]. To enhance the sparsity of the solutions and hence reduce the number of perturbed elements, Eq. (9) can be iteratively minimized as:

$$
\text { minimize }\left\|\beta\left(\boldsymbol{x}^{i-1}\right) \boldsymbol{x}^{i}\right\|_{1} \text { subject to } \boldsymbol{A} \boldsymbol{x}-\boldsymbol{b} \leq \boldsymbol{\varepsilon},
$$

where $\beta\left(\boldsymbol{x}^{i-1}\right)=\frac{1}{\boldsymbol{x}^{i-1}+\delta}, \delta$ is a small positive number that is used to provide stability to array weights, such that the minimization process is assured by estimating non-zero values of the sparse elements and is not affected by the zero values in the next new iterations. The non-zero values of Eq. (10) represent the sparse elements that give exactly the needed number of the perturbed elements. The corresponding array pattern of these perturbed elements can be obtained. Then, the overall array pattern can be computed by:

$$
A F_{\text {proposed }}=A F_{\text {uniform }}-A F_{\text {sparse elements }} .
$$

\section{Simulation Results}

This section demonstrates the performance of the proposed method while generating the required nulls under the minimum number of perturbed elements. In all scenarios, the number of iterations, $i$, and $\delta$ are chosen to be 15 and $10^{-6}$ respectively. 


\subsection{Scenario 1 - Linear Array}

A uniform linear array of $N=20$ elements with interelement spacing equal to $d=0.5 \lambda$ is considered in this scenario. Several cases of null placements are investigated to illustrate the effectiveness of the proposed method. In the first case, a single wide null centered at $49^{\circ}$ is placed.

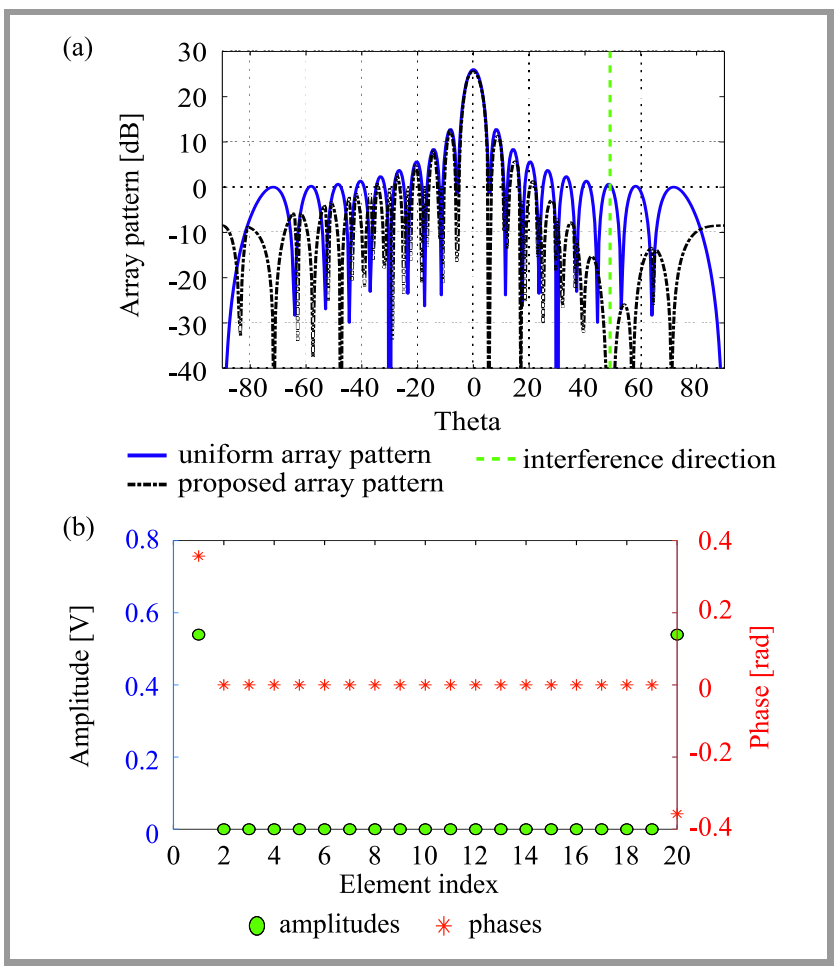

Fig. 1. Radiation patterns of the tested arrays (a) and the corresponding perturbed elements of the proposed array for $N=20$ and a single wide null at $49^{\circ}$ (b). (See digital edition to find the color version).
To obtain such a wide null, two adjacent sharp nulls have to be imposed. For example, to place a wide null centered at $49^{\circ}$ two adjacent sharp nulls are placed at $48.95^{\circ}$ and $49.05^{\circ}$, respectively. Each sharp null needs one perturbed element and, thus, each wide null will need at least two perturbed elements. Further, to achieve such a wide null, it is required that the patterns of the spare array and the uniform array according to Eq. (11) are exactly coincident at the null direction of $49^{\circ}$. Figure 1 shows the results of the proposed array using the compressed sensing approach. The uniform array pattern is also shown for comparison. It can be seen that the required wide null has been successfully placed with only two sparse (non-zero) elements. The perturbed complex weights of these two sparse elements selected randomly by the algorithm are $0.5176+\mathrm{j} 0.2652$ and $0.5176-\mathrm{j} 0.2652$, with indices 1 and 20, respectively.

In the second case, multiple wide nulls are generated. Accordingly, the number of the randomly perturbed elements is expected to increase. However, this increment represents the actual need of the algorithm to perform the required null placement. To highlight this important point, relationship between the required number of nulls and the minimum

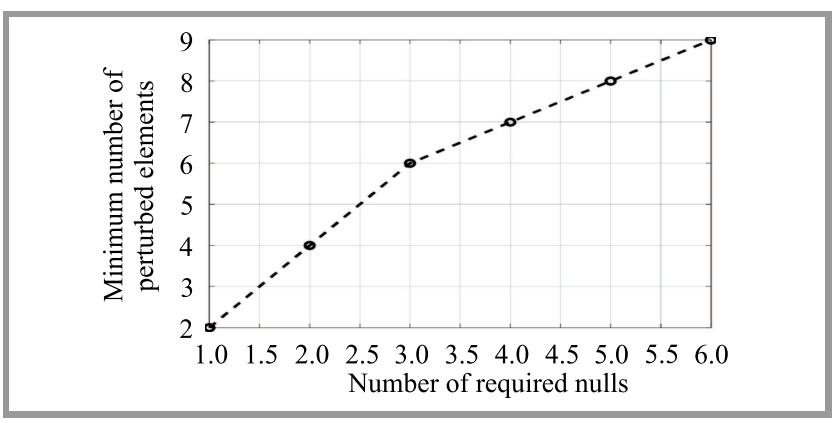

Fig. 2. The minimum number of perturbed elements versus the required number of wide nulls.

Table 1

Design parameters of two and three wide nulls

\begin{tabular}{|c|c|c|c|}
\hline \multicolumn{4}{|c|}{ Two wide nulls } \\
\hline$A$ & & $x$ & $\bar{b}$ \\
\hline \multirow{5}{*}{$\begin{array}{l}4 \times 20 \\
\text { matrix }\end{array}$} & Indices of sparse elements & Complex values of sparse elements & \multirow{5}{*}{$\begin{array}{c}4 \times 1 \text { matrix } \\
-0.1535-\mathrm{j} 0.2222 \\
0.8294+\mathrm{j} 0.1324 \\
0.7756-\mathrm{j} 0.5410 \\
0.3115-\mathrm{j} 0.5702\end{array}$} \\
\hline & 1 & $0.5208+\mathrm{j} 0.3099$ & \\
\hline & 4 & $-0.2612+\mathrm{j} 0.0487$ & \\
\hline & 17 & $-0.2612-\mathrm{j} 0.0487$ & \\
\hline & 20 & $0.5208-\mathrm{j} 0.3099$ & \\
\hline \multicolumn{4}{|c|}{ Three wide nulls } \\
\hline $\bar{A}$ & \multicolumn{2}{|r|}{$x$} & $\bar{b}$ \\
\hline \multirow{7}{*}{$\begin{array}{l}6 \times 20 \\
\text { matrix }\end{array}$} & Indices of sparse elements & Complex values of sparse elements & \multirow{7}{*}{$\begin{array}{c}6 \times 1 \text { matrix } \\
1.4983-\mathrm{j} 0.8935 \\
0.9893-\mathrm{j} 1.6371 \\
0.0153+\mathrm{j} 0.0281 \\
0.3423+\mathrm{j} 0.2908 \\
0.6141-\mathrm{j} 0.3653 \\
0.3006-\mathrm{j} 0.3322\end{array}$} \\
\hline & 1 & $0.2905+\mathrm{j} 0.1139$ & \\
\hline & 5 & $-0.4651-\mathrm{j} 0.2487$ & \\
\hline & 7 & $0.1439+\mathrm{j} 0.3133$ & \\
\hline & 14 & $0.1439-\mathrm{j} 0.3133$ & \\
\hline & 16 & $-0.4651+\mathrm{j} 0.2487$ & \\
\hline & 20 & $0.2905-\mathrm{j} 0.1139$ & \\
\hline
\end{tabular}




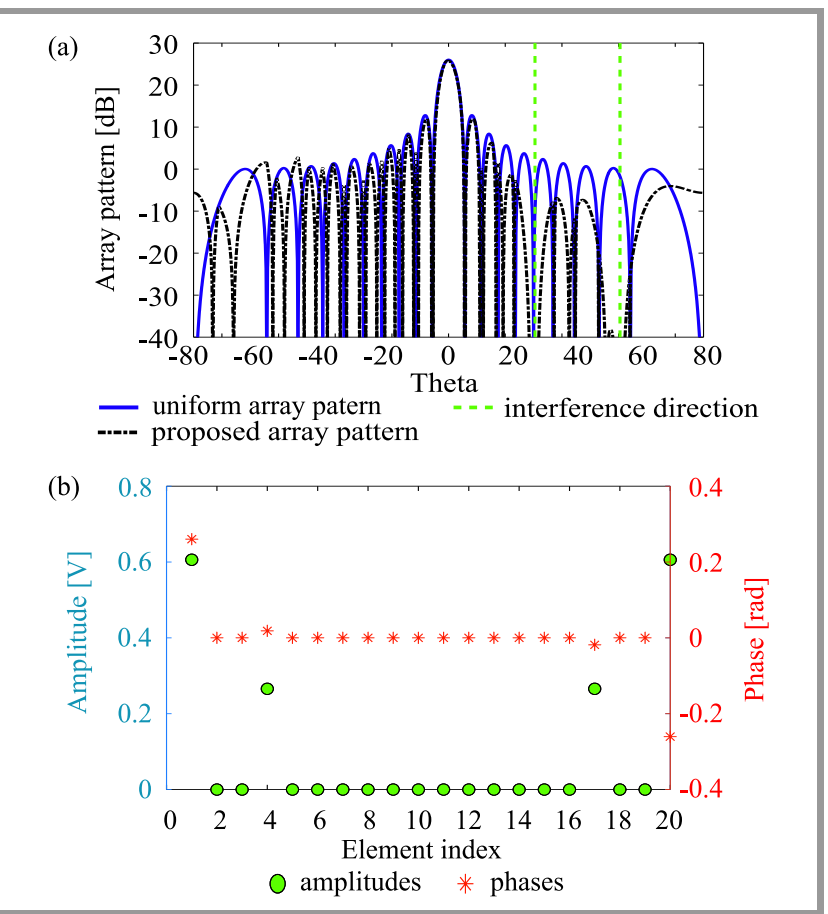

Fig. 3. Radiation patterns of the tested arrays (a) and the corresponding perturbed elements of the proposed array for $N=20$ and two wide nulls at $30^{\circ}$ and $60^{\circ}$ (b).

number perturbed elements are plotted, as shown in Fig. 2. It can be observed that the two wide nulls need at least four perturbed elements. Table 1 shows the complex weights of the sparse elements that are required for generating two and three wide nulls.

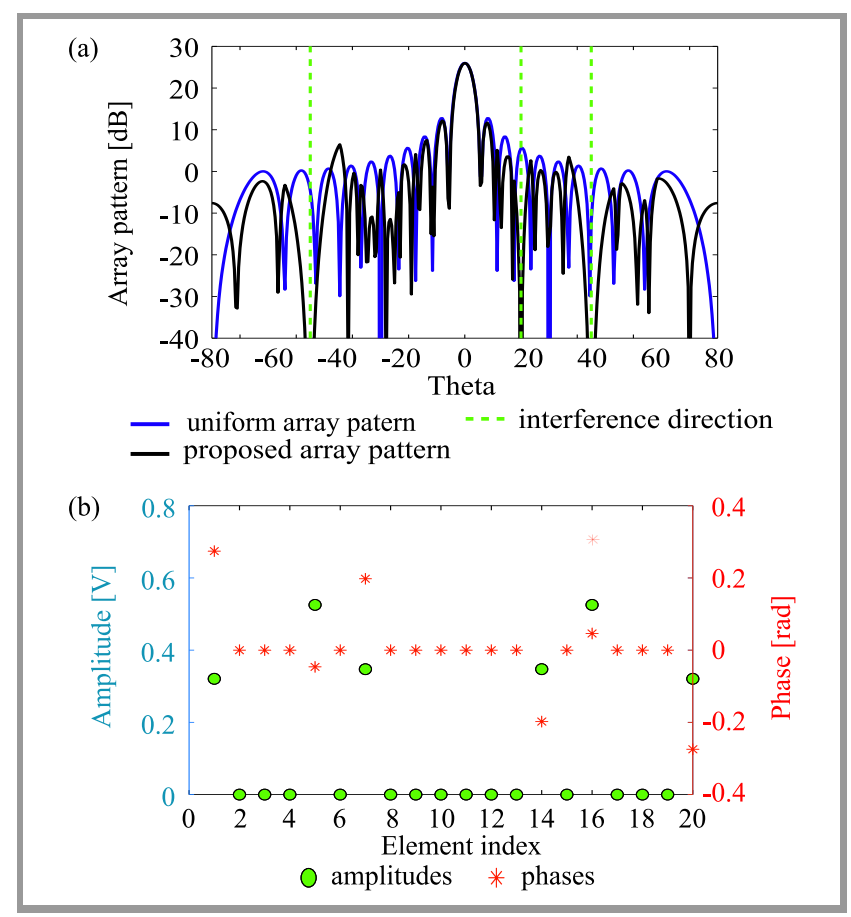

Fig. 4. Radiation patterns of the tested arrays (a) and the corresponding perturbed elements of the proposed array for $N=20$ and three wide nulls at $20^{\circ}, 45^{\circ}$, and $-55^{\circ}$.
Figures 3 and 4 show the results of the tested arrays of two and three wide nulls, respectively. These results fully confirm the effectiveness of the proposed array in placing the required number of wide nulls. Moreover, the directivity of the proposed array is found to be affected only slightly, as long as the number of perturbed elements is lower than the total number of array elements.

\subsection{Scenario 2 - Planar Array}

In this scenario, a square planar array with $20 \times 20$ elements and $\lambda / 2$ inter-element spacing along the $x$ and $y$ axes was considered. In the first use case, the center of the required wide null was chosen to be at $v=-0.5$ and no nulls at $u$ plane were presented, with $v=\sin (\theta) \sin (\varphi)$ and $u=\sin (\theta) \cos (\varphi)$. Figure 5a shows the three-dimensional pattern of the proposed array obtained with the use of the compressed sensing approach, characterized by the minimum number of perturbed elements, while Fig. 5b shows the results of the original uniform planar array shown for comparison purposes.

In the other use case, two wide nulls centered at $v=-0.5$ and $u=-0.7$ are considered. Figure 6 shows the results of this case, with the two required nulls placed successfully.

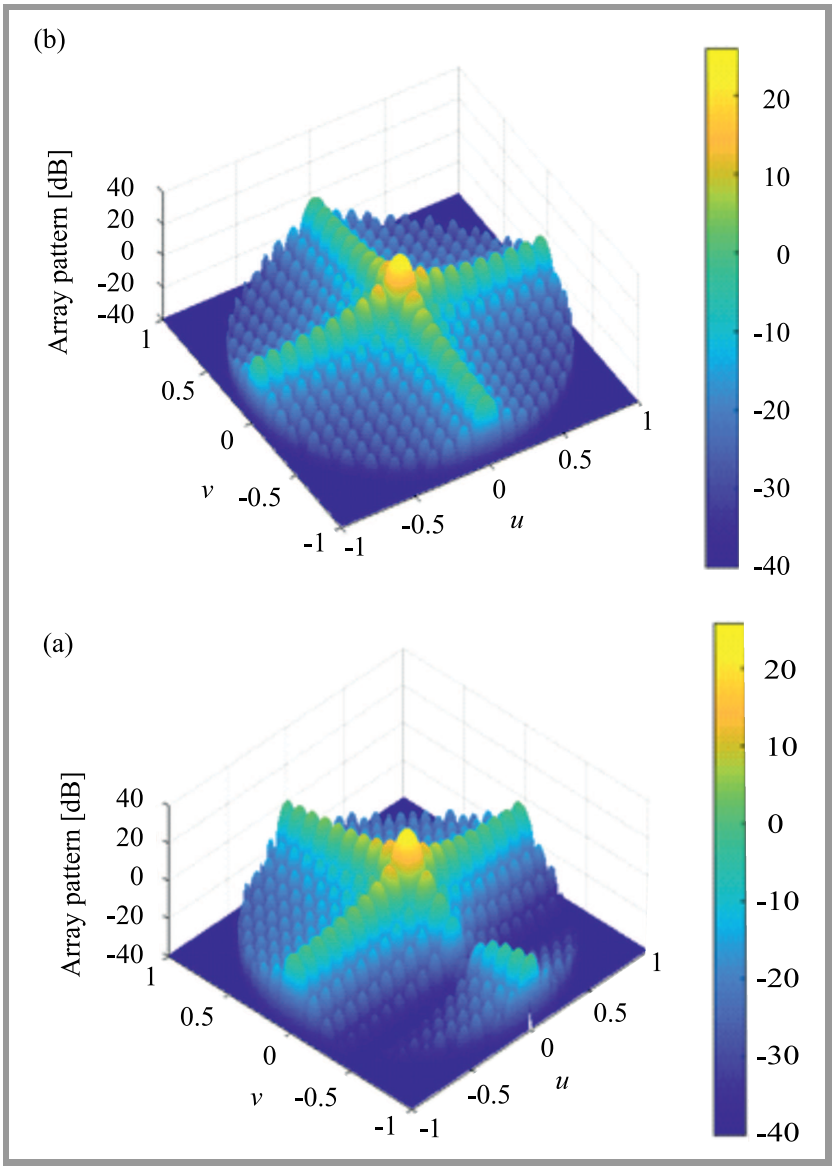

Fig. 5. Radiation patterns of the proposed planar array (a) and the uniform array pattern (b) for $20 \times 20$ and a single wide null centered at $v=-0.5$. 


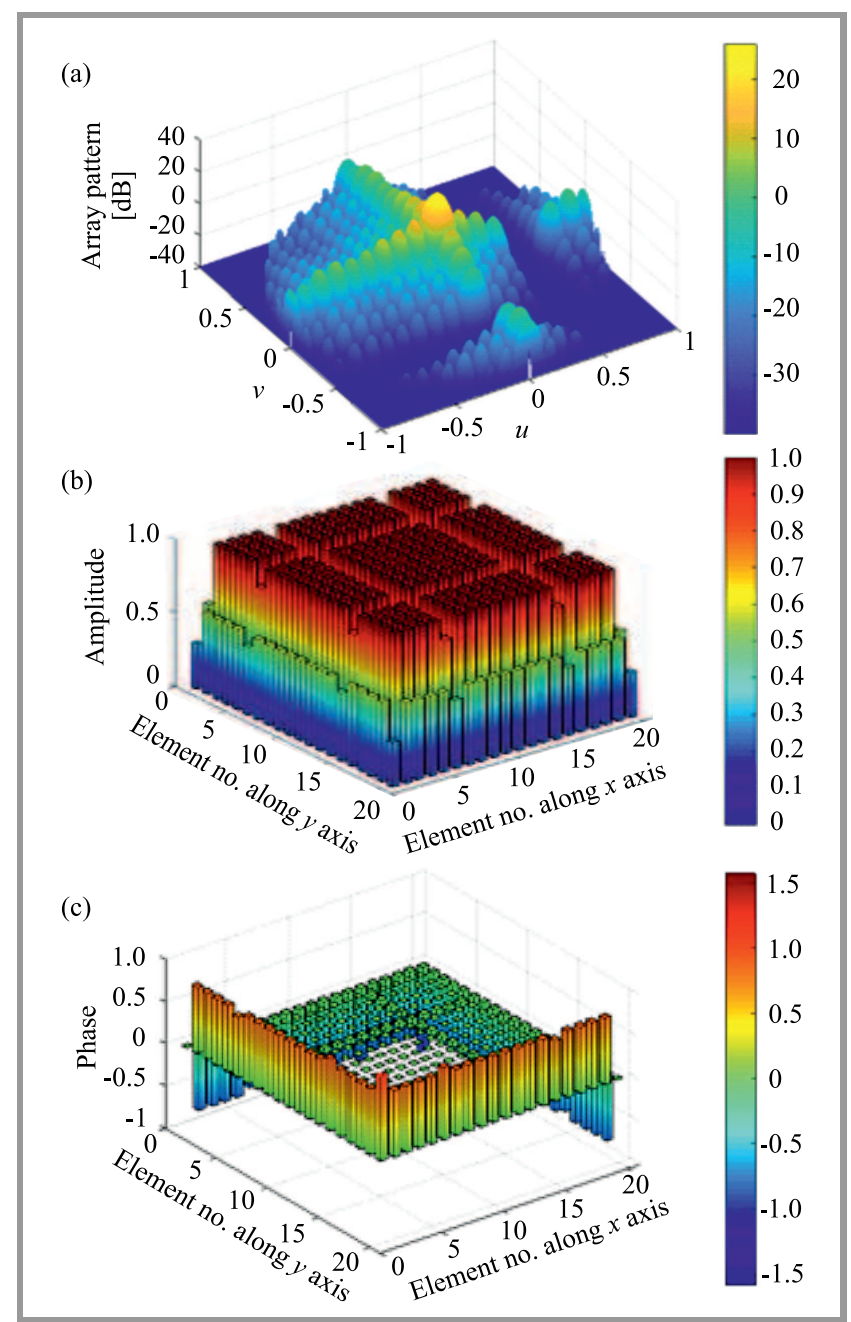

Fig. 6. Radiation patterns of the proposed planar array (a), the corresponding perturbed amplitudes (b), and phases (c).

\section{Conclusions}

An efficient and simple optimization method based on the sparse theory and on the compressed sensing approach was presented for synthesizing linear and planar array patterns with the minimum number of perturbed elements. The simplicity of the proposed method means that the number of the RF components, such as attenuators and phase shifters, is reduced. The proposed array is capable of placing the required wide nulls at undesired directions. For each single wide null there is a need for at least two perturbed elements. Convex programming has been applied to implement and find sparse elements needed to perform the desired null placements. The results show a significant reduction in the complexity of the array feeding network.

\section{References}

[1] M. Fakharzadeh, S. H. Jamali, P. Mousavi, and S. Safavi-Naeini, "Fast beamforming for mobile satellite receiver phased arrays: theory and experiment", IEEE Trans. Antennas Propag., vol. 57, no. 6, pp. 1645-1654, 2009 (DOI: 10.1109/TAP.2009.2019911).
[2] Y. Dong, Z. Chen, P. Fan, and K. B. Letaief, "Mobility-aware uplink interference model for 5G heterogeneous networks", IEEE Trans. Wireless Commun., vol. 15, no. 3, pp. 2231-2244, 2016 (DOI: 10.1109/TWC.2015.2500566).

[3] Z. Geng, H. Deng, and B. Himed, "Adaptive radar beamforming for interference mitigation in radar-wireless spectrum sharing", IEEE Signal Process. Letters, vol. 22, no. 4, pp. 484-488, 2015 (DOI: 10.1109/LSP.2014.2363585).

[4] J. R. Mohammed, "Rectangular grid antennas with various boundary square-rings array", Progress In Electromagnet. Res. Letters, vol. 96, pp. 27-36, 2021 (DOI: 10.2528/PIERL20112402).

[5] J. R. Mohammed, "Obtaining wide steered nulls in linear array patterns by controlling the locations of two edge elements", AEU Int. J. of Electron. and Commun., vol. 101, pp. 145-151, 2019 (DOI: 10.1016/j.aeue.2019.02.004).

[6] C. A. Balanis, Antenna Theory: Analysis and Design, Third Edition, Hoboken, New Jersey: John Wiley \& Sons, 2005, pp. 385-424 (ISBN: 9780471667827).

[7] W. P. M. N. Keizer, "Fast low sidelobe synthesis for large planar array antennas utilizing successive fast Fourier transforms of the array factor", IEEE Trans. Antennas Propag., vol. 55, no. 3, pp. 715722, 2007 (DOI: 10.1109/TAP.2007.891511).

[8] J. R. Mohammed and K. H. Sayidmarie, "Null steering method by controlling two elements", IET Microwaves, Antennas Propag., vol. 8, no. 15, pp. 1348-1355, 2014 (DOI: 10.1049/iet-map.2014.0213).

[9] J. R. Mohammed and K. H. Sayidmarie, "Synthesizing asymmetric sidelobe pattern with steered nulling in non-uniformly excited linear arrays by controlling edge elements", Int. J. of Antennas and Propag., vol. 2017, 2017 (DOI: 10.1155/2017/9293031).

[10] M. Dawoud and M. Nuruzzaman, "Null steering in rectangular planar arrays by amplitude control using genetic algorithms", Int. J. Electron., 2000, vol. 87, no. 12, pp. 1473-1484, 2010 (DOI: 10.1080/00207210050192498).

[11] M. A. Mangoud and H. M. Elragal, "Antenna arrays pattern synthesis and wide null control using enhanced particle swarm optimization", Prog. in Electromag. Res. B, vol. 17, pp. 1-14, 2009 (DOI: 10.2528/PIERB09070205).

[12] A. Trastoy and F. Ares, "Placing quasi-nulls in planar and conformal arrays", Electromagnetics, vol. 19, no. 4, pp. 373-383, 1999 (DOI: 10.1080/02726349908908654).

[13] A. Slowik and H. Kwasnicka, "Evolutionary algorithms and their applications to engineering problems", Neural. Comput. Appl., vol. 32, no. 16 , pp. $12363-12379,2020$ (DOI: 10.1007/s00521-020-04832-8).

[14] J. R. Mohammed, "A new simple adaptive noise cancellation scheme based on ALE and NLMS filter", in Proc. 5th Annual Int. Conf. on Commun. Network and Service Res. (CNSR), Fredericton, New Brunswick, Canada, 2007, pp. 245-254 (DOI: 10.1109/CNSR.2007.4).

[15] U. Singh and M. Rattan, "Design of linear and circular antenna arrays using Cuckoo optimization algorithm", Prog. in Electromag. Res. C, vol. 46, pp. 1-11, 2014 (DOI: 10.2528/PIERC13110902).

[16] S. K. Mahto and A. Choubey, "A novel hybrid IWO/WDO algorithm for nulling pattern synthesis of uniformly spaced linear and non-uniform circular array antenna", AEU Int. J. of Electron. and Commun., vol. 70, no. 6, pp. 750-756, 2016 (DOI: 10.1016/j.aeue.2016.02.013).

[17] P. Saxena and A. Kothari, "Optimal pattern synthesis of linear antenna array using Grey Wolf optimization algorithm", Int. J. Antennas Propag., vol. 2016, pp. 1-11, 2016 (DOI: 10.1155/2016/1205970).

[18] A. J. Abdulkader, J. R. Mohammed, and R. H. Thaher, "Phase-only nulling with limited number of controllable side elements", Prog. in Electromag. Res. C, vol. 99, pp. 167-178, 2020 (DOI: 10.2528/PIERC20010203).

[19] J. R. Mohammed, A. J. Abdulqader, and R. Hamdan, "Antenna pattern optimization via clustered arrays", Prog. in Electromag. Res. M, vol. 95, pp. 177-187, 2020 (DOI: 10.2528/PIERM20042307). 
[20] J. R. Mohammed, A. J. Abdulqader, and R. Hamdan, "Array pattern recovery under amplitude excitation errors using clustered elements", Prog. In Electromag. Res. M, vol. 98, pp. 183-192, 2020 (DOI: 10.2528/PIERM20101906).

[21] J. R. Mohammed, "Thinning a subset of selected elements for null steering using binary genetic algorithm", Prog. in Electromag. Res. $M$, vol. 67, pp. 147-155, 2018 (DOI: 10.2528/PIERM18021604).

[22] J. R. Mohammed and K. H. Sayidmarie, "Performance evaluation of the adaptive sidelobe canceller with various auxiliary configurations", AEU Int. J. of Electron. and Commun., vol. 80, pp. 179-185, 2017 (DOI: 10.1016/j.aeue.2017.06.039).

[23] A. Massa, P. Rocca, and G. Oliveri, "Compressive sensing in electromagnetics - a review", IEEE Antennas Propag. Mag., vol. 57, no. 1, pp. 224-238, 2015 (DOI: 10.1109/MAP.2015.2397092).

[24] J. R. Mohammed, "High-resolution direction-of-arrival estimation method based on sparse arrays with minimum number of elements", J. of Telecommun. and Informat. Technol., vol. 1, pp. 8-14, 2021 (DOI: 10.26636/jtit.2021.143720).

[25] F. Viani, G. Oliveri, and A. Massa, "Compressive sensing pattern matching techniques for synthesizing planar sparse arrays", IEEE Trans. Antennas and Propag., vol. 6, no. 9, pp. 4577-4587, 2013 (DOI: 10.1109/TAP.2013.2267195).

[26] J. Yang and Y. Zhang, "Alternating direction algorithms for 11problems in compressive sensing", SIAM J. on Scien. Comput., vol. 33, no. 1, pp. 250-278, 2011 (DOI: 10.1137/090777761).

[27] H. Mohimani, M. Babaie-Zadeh, and C. Jutten, "A fast approach for over complete sparse decomposition based on smoothed norm", IEEE Trans. Signal Process., vol. 57, no. 1, pp. 289-301, 2009 (DOI: 10.1109/TSP.2008.2007606).

[28] J. A. Tropp and A. C. Gilbert, "Signal recovery from random measurements via orthogonal matching pursuit", IEEE Trans. Inf. Theory, vol. 53, no. 12 , pp. 4655-4666, 2007 (DOI: 10.1109/TIT.2007.909108).

[29] F. Marvasti et al. "A unified approach to sparse signal processing", EURASIP J. Adv. Signal Process., vol. 2012, p. 44, 2012 (DOI: 10.1186/1687-6180-2012-44)

[30] M. A. Abdelhay, N. O. Korany, and S. E. El-Khamy, "Synthesis of uniformly weighted sparse concentric ring arrays based on off-grid compressive sensing framework", IEEE Antenn. and Wireless Prop. Letters, vol. 20, no. 4, pp. 448-452, 2021 (DOI: 10.1109/LAWP.2021.3052174)

[31] M. Khosravi, M. Fakharzadeh, and M. H. Bastani, "Large array null steering using compressed sensing", IEEE Sig. Process. Let., vol. 23, no. 8, pp. 1032-1036, 2016 (DOI: 10.1109/LSP.2016.2580587).

[32] E. J. Candès, M. B. Wakin, and S. P. Boyd, "Enhancing sparsity by reweighted $l_{1}$ minimization", J. Fourier Anal. Appl., vol. 14, no. 5, pp. 877-905, 2008 (DOI: 10.1007/s00041-008-9045-x).

[33] M. Grant and S. Boyd, "CVX: Matlab software for disciplined convex programming, version 2.2", 2020 [Online]. Available: http://cvxr.com/cvx

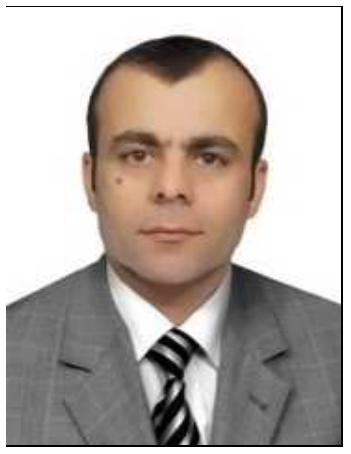

Jafar Ramadhan Mohammed received the B.Sc. and M.Sc. degrees in Electronics and Communication Engineering in 1998, and 2001, respectively, and the Ph.D. degree in Digital Communication Engineering from Panjab University, India in 2009. He was a Visiting Lecturer in the Faculty of Elec- tronics and Computer Engineering at the Malaysia Technical University Melaka (UTeM), Melaka, Malaysia in 2011 and Autonoma University of Madrid, Spain in 2013. He is currently a Professor and Vice Chancellor for Scientific Affairs at Ninevah University. His main research interests are in the area of digital signal processing and its applications, antenna, and adaptive arrays.

(iD) https://orcid.org/0000-0002-8278-6013

E-mail: jafar.mohammed@uoninevah.edu.iq

College of Electronics Engineering

Ninevah University

Mosul, Iraq

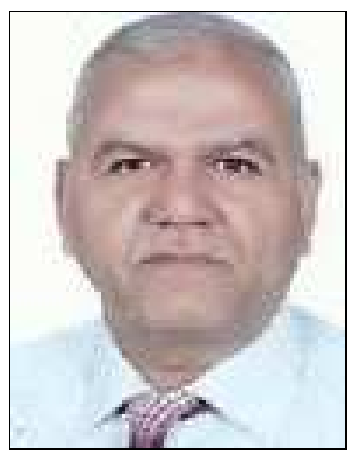

Raad Hamdan Thaher received the M.Sc. degree in Electronics and Communication Engineering in 1981, and the Ph.D. degree in Communication Engineering from Faculty Polytechnic University Bucharest Romania in 1997. He works as a professor in Mustansiriyah University, College of Engineering, Electrical Engineering Department, Baghdad, Iraq. His specialization is in the electronic communication engineering and his research interests are in the field of communication systems, electronics, microwaves, antennas, and communication networks.

E-mail: raadthaher55@gmail.com

Department of Electrical Engineering

Al-Mustansiriya University

Baghdad, Iraq

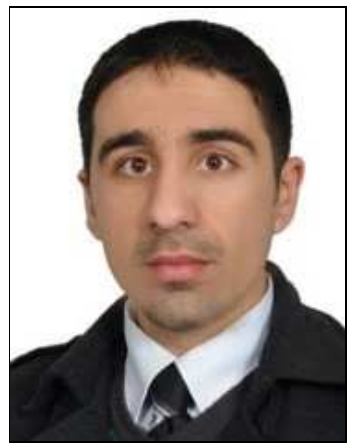

Ahmed Jameel Abdulqader received the B.Sc. and M.Sc. degrees in Electronics and Communication Engineering from the University of Mosul, Iraq, in 2009, and 2013, respectively. He is currently a Ph.D. student at Mustansiriyah University, Baghdad, Iraq. He is Lecturer at Ninevah University. His main research interests are in the area of design and analysis of antenna arrays, array pattern optimization, mobile communication systems, and computer networks.

E-mail: ahmed.abdulqader@uoninevah.edu.iq

College of Electronics Engineering

Ninevah University

Mosul, Iraq 